\title{
Characterization of human cardiac mesenchymal stromal cells and their extracellular vesicles comparing with human bone marrow derived mesenchymal stem cells
}

\author{
In Sook Kang ${ }^{1,2, \#}$, Joowon Suh ${ }^{3, \#}$, Mi-Ni Lee ${ }^{3}$, Chaeyoung Lee, ${ }^{3,4}$ Jing Jin ${ }^{3}$, Changjin Lee ${ }^{5}$, Young Il Yang ${ }^{6}$, Yangsoo Jang ${ }^{1,7} \mathcal{E}^{\circ}$ \\ Goo Taeg $\mathrm{Oh}^{3, *}$ \\ ${ }^{1}$ The Graduate School, Yonsei University College of Medicine, Seoul 03722, ${ }^{2}$ Department of Internal Medicine, Mokdong Hospital, School \\ of Medicine, Ewha Womans University, Seoul 07804, ${ }^{3}$ Department of Life Sciences and College of Natural Sciences, Ewha Womans \\ University, Seoul 03760, Korea, ${ }^{4}$ Faculty of Arts and Science, University of Toronto, Toronto, ON M5S 1A1, Canada, ${ }^{5}$ Rosetta Exosome \\ Inc., Seoul 06159, ${ }^{6}$ Paik Institute for Clinical Research, Inje University College of Medicine, Busan 47392, ${ }^{7}$ Division of Cardiology, \\ Severance Cardiovascular Hospital, Yonsei University College of Medicine, Seoul 03722, Korea
}

Cardiac regeneration with adult stem-cell (ASC) therapy is a promising field to address advanced cardiovascular diseases. In addition, extracellular vesicles (EVs) from ASCs have been implicated in acting as paracrine factors to improve cardiac functions in ASC therapy. In our work, we isolated human cardiac mesenchymal stromal cells (h-CMSCs) by means of three-dimensional organ culture (3D culture) during ex vivo expansion of cardiac tissue, to compare the functional efficacy with human bone-marrow derived mesenchymal stem cells (h-BM-MSCs), one of the actively studied ASCs. We characterized the h-CMSCs as CD90 ${ }^{\text {low }},{\mathrm{c}-\mathrm{kit}^{\text {negative }}}, \mathrm{CD}_{105^{\text {positive }}}$ phenotype and these cells express NANOG, SOX2, and GATA4. To identify the more effective type of EVs for angiogenesis among the different sources of ASCs, we isolated EVs which were derived from CMSCs with either normoxic or hypoxic condition and BM-MSCs. Our in vitro tube-formation results demonstrated that the angiogenic effects of EVs from hypoxia-treated CMSCs (CMSC-Hpx EVs) were greater than the well-known effects of EVs from BM-MSCs (BM-MSC EVs), and these were even comparable to human vascular endothelial growth factor (hVEGF), a potent angiogenic factor. Therefore,

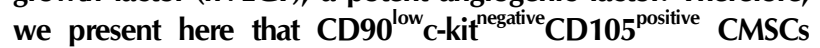
under hypoxic conditions secrete functionally superior EVs for in vitro angiogenesis. Our findings will allow more insights on

${ }^{*}$ Corresponding author. Tel: +82-2-3277-4128; Fax: +82-2-32773760; E-mail: gootaeg@ewha.ac.kr

${ }^{\#}$ These authors contributed equally to this work.

https://doi.org/10.5483/BMBRep.2020.53.2.235

Received 27 September 2019, Revised 28 October 2019, Accepted 28 October 2019

Keywords: Cardiovascular disease, Extracellular vesicles, Mesenchymal stem cell, Regeneration understanding myocardial repair. [BMB Reports 2020; 53(2): 118-123]

\section{INTRODUCTION}

Cardiovascular disease (CVD) is a global major health problem and has remained a leading cause of death, despite the advances in the current medical technologies (1). Cardiovascular regeneration (CVR) with adult stem-cell (ASC) therapy is a promising field for addressing advanced CVD $(2,3)$. Although there are diverse origins of ASCs, the cells that are mostly studied for CVR are mesenchymal stem cells (MSCs) and cardiac stem cells (4). Given the salutary effects of the ASCs for CVD reported by previous studies, there have been many preclinical studies as well as clinical trials conducted with the ASCs $(4,5)$.

The putative 'cardiac stem/progenitor cell (CS/CPC)' is also called a cardiac mesenchymal stem cell, cardiac interstitial cell, cardiac mesenchymal stromal cell (CMSC), or cardiosphere-derived cell (CDC). These cells are usually obtained from the right ventricular septum through cardiac biopsy, or from a left atrial sample of cardiac surgery $(6,7)$. In particular, CDCs indicate the cells derived from cardiospheres; multicellular clusters formed from the primary culture of cardiac specimen. There are several issues about CVR using different types of CS/CPCs. Especially, based on the research that ${\mathrm{c}-\mathrm{kit}^{+}}^{+}$cells were potential CS/CPCs for rejuvenating heart muscles in mice (8), many researchers had considered these cells to be CS/CPCs. However, recent studies have proved that ${\mathrm{C}-\mathrm{kit}^{+}}^{+}$cells are only a small proportion of CDCs, have low regenerative potential, and originate from cardiac endothelial cells, rather than from CS/CPCs $(9,10)$. Another issue is about CD90, which was originally discovered as a thymocyte antigen (thy-1) and can be used as a marker for a variety of stem cells and fibroblasts. Despite limited information regard- 
ing CD90 in CVR, several studies proved that $\mathrm{CD}^{-} \mathrm{CPCs}$ expressed the genes related to stemness and could differentiate into mature cardiomyocytes with complete sarcomere formation $(9,11,12)$. Although these cells also showed a greater regenerative potential in myocardial infarction $(\mathrm{Ml})$, it is still unclear which types of CS/CPCs are more important to the CVR.

Recently, another important concept has been receiving attention in ASC therapy; the paracrine effect. Many studies showed that the cardiac functional advances were not the direct effects of the transplanted CS/CPCs themselves. Instead, such improvement seemed to be induced by means of paracrine factors $(13,14)$; the extracellular vesicle (EV) is one strong candidate (15-17). EVs, also known as exosomes, are nanosized vesicles encompassed by lipid bilayer with diameters from 30-130 nm. EVs exist in almost all biological fluids, including blood, urine, and cell culture medium. They are released from the cells when multivesicular bodies fuse with the cell membrane. They participate as cellular cargos by carrying genetic materials and proteins, facilitate intercellular communication (16), and have specificity that depends on their originating cells (15). EVs from CDCs could inhibit apoptosis, promote proliferation of cardiomyocytes, and improve cardiac functions in $\mathrm{Ml}$ mouse models by means of some miRNAs $(18,19)$.

In this study, we first compared the characterization of h-CMSCs and h-BM-MSCs, and identified EVs from the media of the cells. Relatively few studies have compared the effects of these cells directly (20-22), and especially CD90 ${ }^{\text {low }}$,

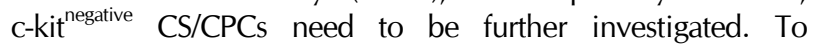
compare the effects of EVs from each cell group, we evaluated in vitro effects of EVs on angiogenesis. Therefore, the

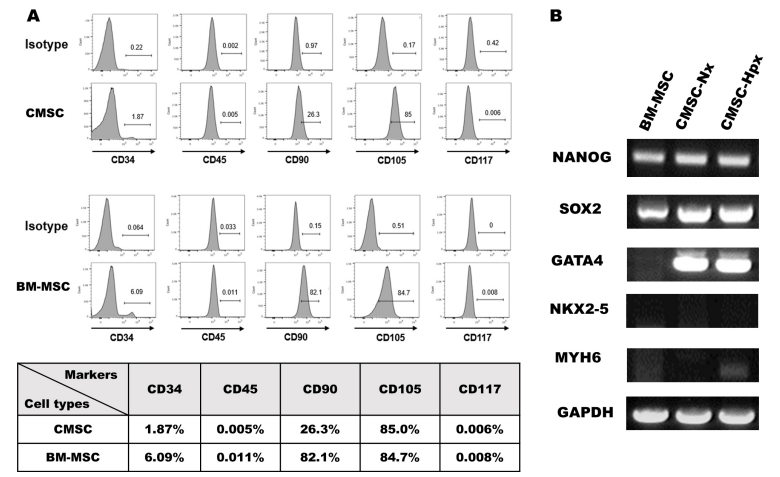

Fig. 1. Characterization of the cells. (A) Flow cytometry results showed negative expression of CD117 and CD45, low expression of $\mathrm{CD} 34$, positive expression of CD105, and different expression of CD90 in the BM-MSCs and CMSCs. (B) RT-PCR analysis showed that the mRNA expression levels of NANOG, SOX2 in BM-MSCs, CMSC-Nx, and CMSC-Hpx were high, whereas the levels were hardly detectable for NKX2-4 and MYH6. The expression of GATA4 was detected only in CMSCs. comparative studies regarding EVs of h-BM-MSCs and h-CMSCs with normoxic and hypoxic conditions would help decide on future effective therapeutic target.

\section{RESULTS}

\section{Characterization of h-BM-MSCs and h-CMSCs}

To characterize the cultured h-BM-MSCs and h-CMSCs, we analyzed various cell surface markers for hematopoietic or cardiac cells by flow cytometry (Fig. 1A). Expression of a cluster of differentiation (CD)45, a type I transmembrane protein that is present in various isoforms on differentiated hematopoietic cells, is less than $1 \%$ in both cell populations. Another hematopoietic stem-cell marker, CD34, is expressed only $1.87 \%$ in CMSCs and $6.09 \%$ in BM-MSCs, which meant that these cells are a CD45 and CD34 negative cell population, as previously reported (23). Importantly, CD90 is differently expressed in each cell population; $82.1 \%$ of BM-MSCs vs. $26.3 \%$ of CMSCs, which is similar to CDCs described (22). Both BM-MSCs and CMSCs express CD105 in about 80\% of the cell population. CD105 (Endoglin), a membrane glycoprotein and a part of the TGF $\beta$ receptor complex, plays a critical role in neoangiogenesis. CD117, known also as c-kit, is a stem-cell growth factor receptor on the surface of hematopoietic stem cells and some cancer cells. Expression levels of CD117 are less than 1\% in both cell groups.

To further define the characteristics of the cells, we did RT-PCR assays for various marker genes. NANOG and SOX2, the transcriptional factors of embryonic stem cells, were expressed in all three cell populations (Fig. 1B). For cardiac markers, mRNAs of NKX2-5 and myosin heavy chain, the $\alpha$ isoform (MYH6), were not noticeable in all groups, but the expression of GATA4, a cardiac-specific transcription factor for proper mammalian cardiac development, was prominent in

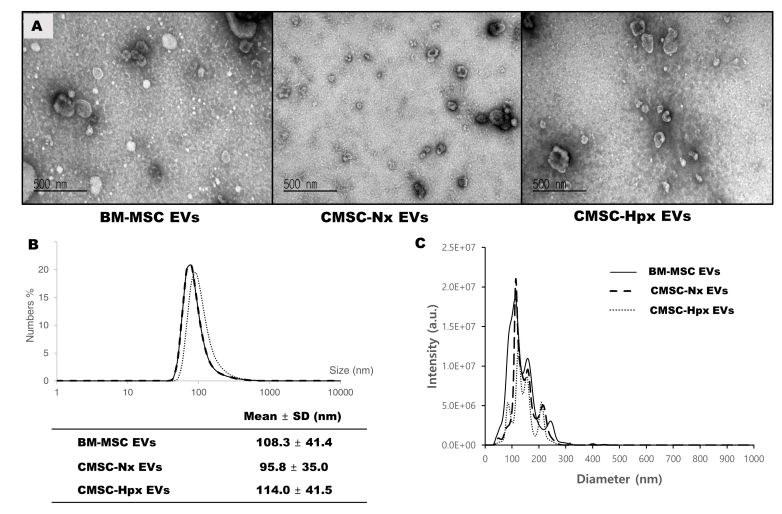

Fig. 2. Morphology and size of EVs. (A) Transmission electron microcopy showed about $100 \mathrm{~nm}$ of round EVs with a cup shape in each group. DLS analysis measured the size distributions (B) and the NTA demonstrated the high particle concentrations corresponding vesicle sizes, and the good purity (C). 
CMSCs but not in BM-MSCs (Fig. 1B). GATA4 and NKX2-5 are early markers of precardiac cells and present synergism as cofactors (24). GATA4 is expressed in CS/CPCs followed by the expression of NKX2-5 and ISL1 of the cardiac mesoderm.

\section{Characterization of EVs isolated from CMSCs and BM-MSCs} EVs were isolated from conditioned media from each cell population. Transmission electron microscopy showed that EVs exhibited umbilicated round morphologies (Fig. 2A). Size measurements by dynamic light-scattering (DLS) analysis found a size of 95-115 nm for EVs (Fig. 2B). CMSC-Hpx EVs are slightly larger than the EVs from normoxia-conditioned CMSCs (CMSC-Nx) (Fig. 2A, B), which was similar to a previous report (21). Nanoparticle tracking analysis (NTA) confirmed the size distribution of EVs and showed sufficient particle concentration (Fig. 2C). The purities of isolated EVs were analyzed by a size exclusion chromatography with high-performance liquid chromatography (HPLC) system, which exhibited levels of contaminating substances smaller than EVs were less than $5 \%$ in all EV preparations (Fig. 3A). Immunoblotting analysis have shown the enrichment of EV surface markers tetraspanins (CD9, CD63, and CD81) with minor differences at expression levels (Fig. 3B), while H2B histone, one of common contaminants in $\mathrm{EV}$ preparation was not detected in all isolated EVs used in this study (Fig. 3B).

\section{CMSC-Hpx EVs exhibit functional superiority on in vitro angiogenesis}

To evaluate the effects of EVs on angiogenesis, we did a tube-formation assay with HUVECs (human umbilical vein endothelial cells). HUVECs were cultured with hVEGF (positive control), BM-MSC EVs, CMSC-Nx EVs, or CMSC-Hpx $\mathrm{EVs}$, and these were compared with the cells with media only (negative control; NC). HUVECs treated with BM-MSC EVs and CMSC-Hpx EVs showed tube formation significantly better than did HUVECs with NC at 6 h (Fig. 4), whereas CMSC-Nx

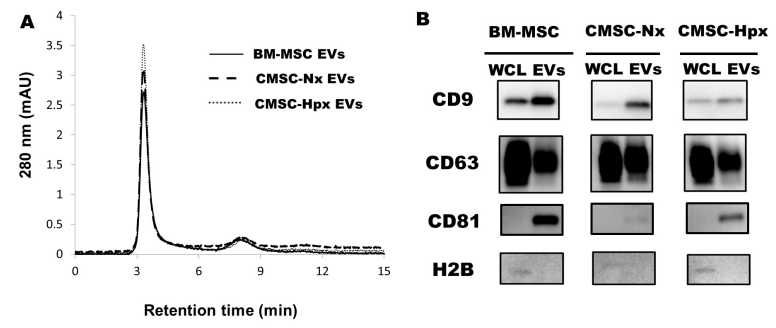

Fig. 3. Purity analysis and markers for EVs. (A) Purity analysis by HPLC showed about $95 \%$ of elutes were within 5 min, which represented EV fractions in BMSCs and CMSCs. The small peak at 8 min contains substances with small molecular weight. (B) Immunoblotting analysis against EV surface markers, CD9, CD63, and CD81 on EV groups and whole cell lysates. H2B, histone, one of common contaminating substance in EV preparation, was not detected in all isolated EVs.
EVs failed to demonstrate superiority over NC in tube formation. The formation of tube-like structures was increased with time up to $6 \mathrm{~h}$ in all groups of experiments. Interestingly, the tube-like structures lasted for $8 \mathrm{~h}$ in the cells treated with VEGF or CMSC-Hpx, whereas these structures started to collapse around $8 \mathrm{~h}$ in the group of BM-MSC EVs (Fig. 4B, C). With our results, CMSC-Nx EVs did not show improvement in tube formation, and only CMSC-Hpx EVs were able to improve the tube formation, creating tube-like structures that persisted for $8 \mathrm{~h}$, all of which were comparable to the effect of VEGF.

\section{DISCUSSION}

We used the CMSCs obtained by ex vivo expansion of human heart tissue using 3D culture as previously described (25). These CMSCs did not form cardiospheres, but shared similar phenotypes with CDCs of $\mathrm{CD}^{\text {low }}, \mathrm{c}^{\mathrm{k}} \mathrm{kit}^{\text {negative }}, \mathrm{CD} 105^{\text {positive }}$. The CMSCs were distinguished from MSCs in the expression of CD90 (22).

Recently, a decision has been made on an issue concerning the regeneration of CS/CPCs, especially c-kit ${ }^{+}$cells $(6,8,26)$ : 31 papers from a researcher at the Harvard lab studying the role of ${\mathrm{c}-\mathrm{kit}^{+}}^{+}$cells as potential CS/CPCs were retracted

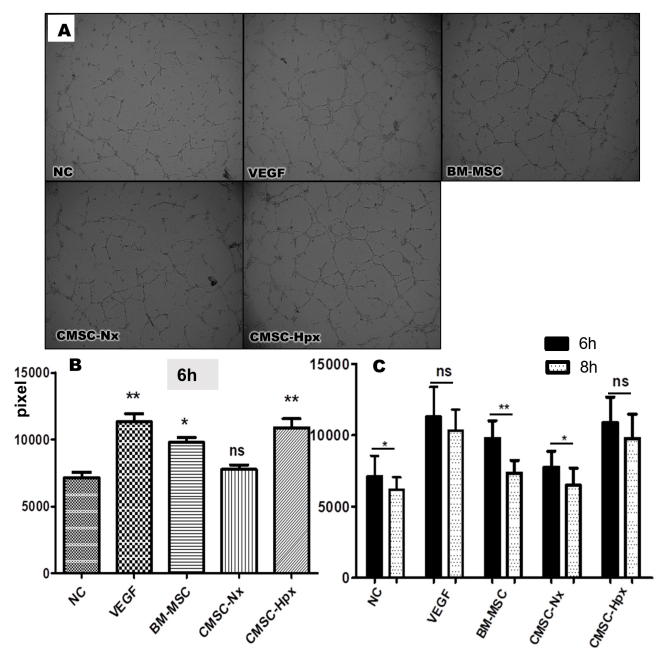

Fig. 4. Tube-formation assay of HUVECs treated with three types of EVs. (A) Microscopy images were taken for the tube-formation assay of HUVECs at $6 \mathrm{~h}$. Endothelial growth media was used for a negative control (NC) and VEGF $(20 \mathrm{ng} / \mathrm{ml})$ was used for positive control; Cells were treated with media including $\mathrm{EVs}$ from BM-MSCs, CMSC-Nx, or CMSC-Hpx for experiments. Detailed methods are described in the supplemental materials. (B) Total tube length at $6 \mathrm{~h}$ showed the augmented tube formation in the groups treated with VEGF, BM-MSC EVs, or CMSC-Hpx EVs; *P $<0.01,{ }^{* * P}<0.001$ vs. NC, ns = no significance; ANOVA followed by Turkey post hoc analysis. (C) The tube-like structure was sustained until $8 \mathrm{~h}$ in VEGF and CMSC-Hpx EVs; ${ }^{*} \mathrm{P}<$ $0.05, * * P<0.01$; student t-test within group at $6 \mathrm{~h}$ and $8 \mathrm{~h}$. 
because of 'the inclusion of fabricated and/or falsified data' and the ongoing clinical trials involving these cells were paused $(27,28)$. Many scientists say that it is time to reset the putative $\mathrm{CS} / \mathrm{CPC}$ research, although there are some researchers saying that $\mathrm{c}-\mathrm{kit}^{+}$cells are still valid for further analysis of regeneration effects based on some animal study results (28, 29). However, it was known that c-kit ${ }^{+}$cells are only a minor fraction of the whole CDC from previous reports (22), and these cells barely differentiate into cardiomyocytes. Moreover, the remnant $\mathrm{c}-\mathrm{kit}^{+}$cells in the heart are regarded as cardiac endothelial cells rather than as CS/CPCs (30). Retrospective analysis of a recently completed clinical trial (CADUCEUS) demonstrated that c-kit expression was not related to the therapeutic effect of CDCs in humans, and that $\mathrm{c}-\mathrm{kit}^{+}$cells were functionally inferior to unsorted human CDCs (9). Given these previous studies, our study tested the idea that CS/CPCs with low c-kit expression may secrete paracrine factors by means of EVs to promote angiogenesis. In our study, both BM-MSCs and CMSCs hardly expressed c-kit.

Meanwhile, CMSCs and BM-MSCs are distinguished by CD90 expression, and unlike BM-MSCs, CMSCs expressed only $26 \%$ of CD90. Li et al. also reported a similar profile of gene expression: the expression of CD90 as $18.4 \%$ in CDCs, compared with an expression of $99.0 \%$ in BM-MSCs (22). Thy-1 knock-out mice are viable and grossly normal (31), and the definite roles of $\mathrm{CD}^{-}$clones in CVR are not clear, but some researchers reported data regarding this topic. They demonstrated the superiority of $\mathrm{CD}^{-} 0^{-} \mathrm{CDCs}$ over $\mathrm{CD} 90^{+}$ CDCs $(9,11,12)$. In the chronic Ml rat model, injection of $\mathrm{CD}^{-}{ }^{-}$CMSCs augmented cardiac function outperforming $\mathrm{CD}^{+}$cells, and histological analysis of $\mathrm{CD}^{-}$cells revealed an increase in vascularization within the infarct lesion (12). Other studies reported similar salutary effects, since CD90 ${ }^{-}$cells secret less inflammatory cytokines and may differentiate into cardiomyocytes (9). Interestingly, CD90cells expressed significantly more hepatocyte growth factor (HGF) than did $\mathrm{CD}^{+} 0^{+}$cells, and both $\mathrm{CD}^{-}$and $\mathrm{CD}^{+}$ CMSCs secrete VEGF or basic fibroblast growth factor (bFGF) comparably (12).

By means of the transcriptional profiles and gene ontology enrichment analysis of adult human CDCs subdivided by the expression of CD90, CD90 ${ }^{-}$clones were enriched with the genes related to stemness, whereas $\mathrm{CD}^{+} 0^{+}$clones were enriched with fibroblast-associated genes (11). Two separate studies found that the expression level of CD90/THY-1 changes dynamically at the formation of cardiospheres under different culture conditions $(11,32)$. Our CMSCs were isolated as $\mathrm{CD} 90^{\text {low }} \mathrm{CS} / \mathrm{CPCs}$ under specific culture condition to promote stemness (25).

Hence, our $\mathrm{CD} 90^{\text {low }} \mathrm{C}-\mathrm{kit}^{\text {negative }} \mathrm{CD} 105^{\text {positive }} \mathrm{CMSC}$ are likely to play a significant role in the regeneration of the heart as an active fraction. Most research with CS/CPCs has been done with CDCs of heterogeneous populations or with c-kit ${ }^{+}$cells, so far. Therefore, further studies with ${\mathrm{c}-k \mathrm{kit}^{\text {negative }}}$ and
$\mathrm{CD}^{\text {negative }}$ clones are needed to reveal the more effective therapeutic application of CS/CPC and would be helpful for understanding the roles of these cells.

GATA4 is one of the earliest transcription factors and a critical regulator of cardiac development, and interacts with many transcriptional factors, such as NKX2-5 (33, 34). Although GATA4 is crucial for regulating the expression of NKX2-5, it has been proposed that additional signaling pathways are needed for the initial activation of NKX2-5 (34, 35). In our results, CMSCs expressed only GATA4, but not NKX2-5. Other studies using CS/CPCs from rotating tissue-culture conditions similar to our work, have also reported that GATA4 is expressed with limited expression of NKX2-5, which proposed these cells in early developmental stages $(11,36)$. Therefore, our cells may also be considered to be in early developmental stages before expressing NKX2-5, and further analysis will help us understand the roles of CS/CPCs at various developmental stages or marking various kinds of stemness during primary culture.

Our results showed that CMSC-Hpx EVs improved tube formation, but CMSC-Nx EVs did not. EV release from hypoxic MSCs was associated with an increased hypoxia-inducible factor 1-alpha activation, which could increase angiogenesis through Jagged-1 $(37,38)$. Hypoxia-treated EVs from human adipose-derived MSCs were reported to activate the protein kinase A signaling pathway and promote the expression of VEGF (20). EVs from hypoxic CDCs (CD90 high cells) were reported to contain the increased level of EV-assocoated miRNAs and improved the tube formation of HUVECs (21). A study using EVs from CPC-Hpx $\left({\left.\mathrm{c}-k i t^{+}\right)}^{+}\right.$reported the improvement of rat cardiac function and hypertrophy (14). These imply that tube formation of $\mathrm{CD} 0^{\text {low }} \mathrm{C}-\mathrm{kit}^{\text {negative }} \mathrm{CMSC}$ EVs under hypoxia was increased by triggering such signaling pathways. Further studies with our $\mathrm{CD} 90^{\text {low }} \mathrm{C}-\mathrm{kit}^{\text {negative }} \mathrm{CMSCs}$ will add valuable insights on understanding the extracellular vesicular influence of these cells in hypoxic conditions. The clinical potential of EVs could greatly depend on the cell-culture environment. Further research is needed to examine how CMSCs under hypoxic conditions could produce more effective EVs. In earlier works, growth factors including HGF, VEGF, and bFGF were reported to be related to these functional differences between CDCs and MSCs (22) and between the $\mathrm{CD}^{-} 0^{-}$and $\mathrm{CD} 90^{+} \mathrm{CDCs}$ (12). Since EVs have been reported to contain various growth factors, it is very likely that the more effective function of CMSC-Hpx EVs in our study resulted from the secretion of EVs with growth factors like HGF.

There are several questions to be answered by future research with EVs from CMSCs. First, the yield of EVs from CMSCs was lower than the yield of BM-MSC EVs (Supplementary Fig. 1). CMSC-EV tended to be more clingy, possibly because of the different nature of EVs, than were BM-MSC EVs, which may have caused unpredictable frequent loss during the EV isolation. Therefore, it was difficult to 
obtain sufficient EVs from CMSCs of our limited human samples. These technical problems should be solved for further experiments.

Second, the degree of tube formation was not increased in proportion to the concentration of EVs. Instead, the effect was best at the dose of $2 \mu \mathrm{g} / \mathrm{ml}(0.4 \mu \mathrm{g} /$ well $)$ of EVs in all three groups, but was decreased with a higher concentration of EVs (Supplementary Fig. 2). There have been reports of biphasic results of tube augmentation with increasing concentration of EVs, although others demonstrated that angiogenesis increases dose-dependently with EVs treatment $(21,39)$. Additionally, they used ten times more EV concentration than were used in our work $(25 \mu \mathrm{g} / \mathrm{ml}$ versus $2 \mu \mathrm{g} / \mathrm{ml})$. These differences between different laboratories could have resulted for various technical reasons, such as different isolation methods, the purity of EVs, and different cell-culture environments. Controlling the detailed cell-culture condition and the standardized isolation methods of EVs are needed to solve the quality and quantity differences of EVs.

Our study showed that the hypoxic condition of $\mathrm{CD} 0^{\text {low }} \mathrm{c}-\mathrm{kit}^{\text {negative }} \mathrm{CMSCs}$ could increase the secretion of EVs with active components for angiogenesis. These imply the functional importance of $\mathrm{CD} 90^{\text {low }} \mathrm{C}$-kit ${ }^{\text {negative }} \mathrm{CMSCs}$, which has often been underestimated in the CVD. Therefore, our results would help us understand the role of $\mathrm{CD} 90^{\text {low }} \mathrm{c}-\mathrm{kit}^{\text {negative }}$ cardiac cells and apply their EVs clinically to cardiac regeneration after CVD. Further investigations are needed to define the mechanism and the functional characterization of CMSC-Hpx EVs.

\section{MATERIALS AND METHODS}

Details are provided in the Supplementary materials.

\section{Human CMSCs preparation}

CMSCs were explanted from cadaveric tissue and expanded as described previously (25). Briefly, after $14 \mathrm{~d}$ of dynamic tissue culture, outgrown cells were collected and suspended in growth-culture media for a conventional monolayer culture condition. When cells reached $80 \%$ confluence, they were detached and subcultured. Experiments were approved by the institutional review board of Busan Paik Hospital (IRB No. 2016-11-0006).

\section{ACKNOWLEDGEMENTS}

This research was supported by a grant from the Korean Health Technology R\&D Project through the Korean Health Industry Development Institute (KHIDI), funded by the Ministry of Health \& Welfare, Republic of Korea (grant number: HI15C1277).

\section{CONFLICTS OF INTEREST}

The authors have no conflicting interests.

\section{REFERENCES}

1. Benjamin EJ, Muntner P, Alonso A et al (2019) Heart Disease and Stroke Statistics-2019 Update: A Report From the American Heart Association. Circulation 139, e56-e528

2. Cambria E, Pasqualini FS, Wolint $P$ et al (2017) Translational cardiac stem cell therapy: advancing from first-generation to next-generation cell types. NPJ Regen Med 2, 17

3. Makkar RR, Smith RR, Cheng K et al (2012) Intracoronary cardiosphere-derived cells for heart regeneration after myocardial infarction (CADUCEUS): a prospective, randomised phase 1 trial. Lancet 379, 895-904

4. Parizadeh SM, Jafarzadeh-Esfehani R, Ghandehari $M$ et al (2019) Stem cell therapy: A novel approach for myocardial infarction. J Cell Physiol 234, 16904-16912

5. Yun CW and Lee SH (2019) Enhancement of Functionality and Therapeutic Efficacy of Cell-Based Therapy Using Mesenchymal Stem Cells for Cardiovascular Disease. Int J Mol Sci 20, 982

6. Barile L, Messina E, Giacomello A and Marban E (2007) Endogenous cardiac stem cells. Prog Cardiovasc Dis 50, 31-48

7. Smith RR, Barile L, Cho HC et al (2007) Regenerative potential of cardiosphere-derived cells expanded from percutaneous endomyocardial biopsy specimens. Circulation 115, 896-908

8. Beltrami AP, Barlucchi L, Torella D et al (2003) Adult Cardiac Stem Cells Are Multipotent and Support Myocardial Regeneration. Cell 114, 763-776

9. Cheng K, Ibrahim A, Hensley MT et al (2014) Relative roles of CD90 and c-kit to the regenerative efficacy of cardiosphere-derived cells in humans and in a mouse model of myocardial infarction. J Am Heart Assoc 3, e001260

10. Gude NA and Sussman MA (2018) Chasing c-Kit through the heart: Taking a broader view. Pharmacol Res 127, 110-115

11. Gago-Lopez N, Awaji O, Zhang Y et al (2014) THY-1 receptor expression differentiates cardiosphere-derived cells with divergent cardiogenic differentiation potential. Stem Cell Reports 2, 576-591

12. Shen D, Shen M, Liang $H$ et al (2018) Therapeutic benefits of CD90-negative cardiac stromal cells in rats with a 30-day chronic infarct. J Cell Mol Med 22, 1984-1991

13. Eschenhagen T, Bolli R, Braun $T$ et al (2017) Cardiomyocyte Regeneration. Circulation 136, 680-686

14. Agarwal U, George A, Bhutani S et al (2017) Experimental, Systems, and Computational Approaches to Understanding the MicroRNA-Mediated Reparative Potential of Cardiac Progenitor Cell-Derived Exosomes From Pediatric Patients. Circ Res 120, 701-712

15. Bei Y, Das S, Rodosthenous RS et al (2017) Extracellular Vesicles in Cardiovascular Theranostics. Theranostics 7, 
4168-4182

16. Ibrahim A and Marban E (2016) Exosomes: Fundamental Biology and Roles in Cardiovascular Physiology. Annu Rev Physiol 78, 67-83

17. Zamani P, Fereydouni N, Butler AE, Navashenaq JG and Sahebkar A (2018) The therapeutic and diagnostic role of exosomes in cardiovascular diseases. Trends Cardiovasc Med 29, 313-323

18. Ibrahim Ahmed GE, Cheng K and Marbán E (2014) Exosomes as Critical Agents of Cardiac Regeneration Triggered by Cell Therapy. Stem Cell Reports 2, 606-619

19. Sahoo S and Losordo DW (2014) Exosomes and cardiac repair after myocardial infarction. Circ Res 114, 333-344

20. Xue C, Shen Y, Li X et al (2018) Exosomes Derived from Hypoxia-Treated Human Adipose Mesenchymal Stem Cells Enhance Angiogenesis Through the PKA Signaling Pathway. Stem Cells Dev 27, 456-465

21. Namazi H, Mohit E, Namazi I et al (2018) Exosomes secreted by hypoxic cardiosphere-derived cells enhance tube formation and increase pro-angiogenic miRNA. J Cell Biochem 119, 4150-4160

22. Li TS, Cheng K, Malliaras K et al (2012) Direct comparison of different stem cell types and subpopulations reveals superior paracrine potency and myocardial repair efficacy with cardiosphere-derived cells. J Am Coll Cardiol 59, 942-953

23. Maleki M, Ghanbarvand F, Reza Behvarz M, Ejtemaei M and Ghadirkhomi E (2014) Comparison of mesenchymal stem cell markers in multiple human adult stem cells. Int J Stem Cells 7, 118-126

24. Durocher D, Charron F, Warren R, Schwartz RJ and Nemer M (1997) The cardiac transcription factors Nkx2-5 and GATA-4 are mutual cofactors. EMBO J 16, 5687-5696

25. Kim JT, Chung HJ, Seo JY et al (2015) A fibrin-supported myocardial organ culture for isolation of cardiac stem cells via the recapitulation of cardiac homeostasis. Biomaterials 48, 66-83

26. van Berlo JH, Kanisicak O, Maillet M et al (2014) c-kit+ cells minimally contribute cardiomyocytes to the heart. Nature 509, 337-341

27. G K Harvard calls for retraction of dozens of studies by noted cardiac researcher. https://www.nytimes.com/2018/ 10/15/health/piero-anversa-fraud-retractions.html. Published October 15, 2018. Accessed September 25,
2019.

28. Kaiser J (2018) Suspect science leads to pause in stem cell trial. Science 362, 513

29. Epstein JA (2019) A Time to Press Reset and Regenerate Cardiac Stem Cell Biology. JAMA Cardiol 4, 95-96

30. Sultana N, Zhang L, Yan J et al (2015) Resident c-kit(+) cells in the heart are not cardiac stem cells. Nat Commun 6, 8701

31. Rege TA and Hagood JS (2006) Thy-1, a versatile modulator of signaling affecting cellular adhesion, proliferation, survival, and cytokine/growth factor responses. Biochim Biophys Acta 1763, 991-999

32. Forte E, Miraldi F, Chimenti I et al (2012) TGFbeta-dependent epithelial-to-mesenchymal transition is required to generate cardiospheres from human adult heart biopsies. Stem Cells Dev 21, 3081-3090

33. Heikinheimo M, Scandrett JM and Wilson DB (1994) Localization of transcription factor GATA-4 to regions of the mouse embryo involved in cardiac development. Dev Biol 164, 361-373

34. Yilbas AE, Hamilton A, Wang $Y$ et al (2014) Activation of GATA4 gene expression at the early stage of cardiac specification. Front Chem 2, 12

35. Akazawa $\mathrm{H}$ and Komuro I (2005) Cardiac transcription factor Csx/Nkx2-5: Its role in cardiac development and diseases. Pharmacol Ther 107, 252-268

36. Oldershaw R, Owens WA, Sutherland R et al (2019) Human Cardiac-Mesenchymal Stem Cell-Like Cells, a Novel Cell Population with Therapeutic Potential. Stem Cells Dev 28, 593-607

37. Lo Sicco C, Reverberi D, Balbi C et al (2017) Mesenchymal stem cell-derived extracellular vesicles as mediators of anti-inflammatory effects: endorsement of macrophage polarization. Stem Cells Transl Med 6, 1018-1028

38. Hernán GK, Nahuel AG, Imelda O et al (2017) Hypoxia inducible factor-1apotentiates jagged1-mediated angiogenesis by mesenchymal stemcell-derived exosome. Stem Cells 35, 1747-1759

39. Gray WD, French KM, Ghosh-Choudhary S et al (2015) Identification of therapeutic covariant microRNA clusters in hypoxia-treated cardiac progenitor cell exosomes using systems biology. Circ Res 116, 255-263 